\title{
A NOTE ON THE TWO CARDINAL PROBLEM
}

\author{
C. C. CHANG ${ }^{1}$
}

In this note we prove a theorem concerning the two cardinal problem (see [1], [4], [6], [7] for reference and for some of the standard notation); this result has been referred to in $[6$, p. 311] and [7, (3.7) ]. The problem, first proposed by Vaught, is as follows. Let $T$ be a first-order theory and let $U$ be a unary predicate symbol in the language of $T . T$ is said to admit the pair $\alpha, \beta$ of cardinals if there exists a model $M=\langle A, U, S, \cdots\rangle$ of $T$ such that $|A|=\alpha$ and $|U|=\beta$. Suppose $T$ admits a pair $\alpha, \beta$ where $\alpha>\beta \geqq \omega$. Then what other pairs of cardinals $\gamma, \delta$ must $T$ admit? The following theorem gives a partial answer.

THEOREM. Let $T$ be a theory in a countable first-order language $L$ with identity. Assume the generalized continuum hypothesis. If $T$ admits a pair $\alpha, \beta$ where $\alpha>\beta \geqq \omega$, then $T$ admits all pairs $\delta^{+}, \delta$ where $\delta$ is a regular infinite cardinal.

Proof. We first note that in the case $\delta=\omega$ the theorem is already known [4, Theorem 6.2], and in fact this particular case can be proved without assuming the generalized continuum hypothesis. Henceforth we assume that $\delta$ is a regular infinite cardinal greater than $\omega$.

Let us suppose that $L$ has, in addition to the unary predicate symbol $U$, some other predicate symbols $S, \cdots$, and, for the sake of definiteness, let us assume that $\boldsymbol{S}$ is a ternary predicate symbol. Let

$$
\langle A, U, S, \cdots\rangle
$$

be a model of $T$ such that $|A|=\alpha$ and $|U|=\beta$, where $\alpha>\beta \geqq \omega$. Let $R$ be a new binary relation over $A$ such that $R \subset U^{2}$ and $R$ indexes all the finite subsets of $U$. That is,

(1) for any $u_{1}, \cdots, u_{n} \in U$, there exists a $u \in U$ such that for all $t \in U$,

$$
R(u, t) \text { if and only if } t \in\left\{u_{1}, \cdots, u_{n}\right\} .
$$

It is always possible to construct such a relation $R$ as $U$ is infinite.

Received by the editors July 27, 1964.

1 This work was partly supported by an NSF research grant and by an NSF Senior Postdoctoral Fellowship held at the Institute for Advanced Study, Princeton, during 1962-1963. 
Let $L_{1}$ be the first-order language obtained from $L$ by adding the new binary predicate symbol $\boldsymbol{R}$. The model

$$
\langle A, U, R, S, \cdots\rangle
$$

is then a model for $L_{1}$. In view of the downward Löwenheim-Skolem theorem as formulated in [5], and since $L_{1}$ is still countable, there exists a subset $B \subset A$ such that

$$
\begin{aligned}
& U \subset B,|B|=\beta, \text { and } \\
& \quad\left\langle B, U, R, S \cap B^{3}, \cdots\right\rangle \prec\langle A, U, R, S, \cdots\rangle .
\end{aligned}
$$

Let $L_{2}$ be the first-order language obtained from $L_{1}$ by adding the new unary predicate symbol $B$. Thus

$$
\langle A, B, U, R, S, \cdots\rangle
$$

is a model for $L_{2}$. Let $T^{\prime}$ be the complete theory given by $\langle A, B, U, R, S, \cdots\rangle$. We note in passing that, by (1), $T^{\prime}$ contains in particular all closures of formulas of the form

$$
\begin{aligned}
U\left(z_{1}\right) & \wedge \cdots \wedge U\left(z_{m}\right) \wedge \phi\left(z_{1}\right) \wedge \cdots \wedge \phi\left(z_{m}\right) \\
& \rightarrow(\exists y)\left(R\left(y, z_{1}\right) \wedge \cdots \wedge R\left(y, z_{m}\right) \wedge(\forall t)(R(y, t) \rightarrow \phi(t))\right),
\end{aligned}
$$

where $\phi$ is an arbitrary formula of $L_{2}$ which may contain other free variables.

Clearly $T^{\prime}$ is consistent and has an infinite model. It is well known that, assuming the generalized continuum hypothesis, for each regular infinite cardinal $\delta>\omega, T^{\prime}$ has a $\delta$-saturated model (see, for example, $[4],[3]$ for some properties of saturated models)

$$
N_{0}=\left\langle A_{0}, B_{0}, U_{0}, R_{0}, S_{0}, \cdots\right\rangle
$$

of power $\delta$. By (2), both $B_{0}$ and $U_{0}$ must be infinite, and since $N_{0}$ is $\delta$-saturated, it follows that $\left|B_{0}\right|=\left|U_{0}\right|=\delta$. Furthermore, there are enough sentences in $T^{\prime}$ to insure that the model (in $L_{1}$ )

$$
N_{1}=\left\langle B_{0}, U_{0}, R_{0}, S_{0} \cap\left(B_{0}\right)^{3}, \cdots\right\rangle
$$

is a proper elementary submodel of the model (in $L_{1}$ )

$$
M_{0}=\left\langle A_{0}, U_{0}, R_{0}, S_{0}, \cdots\right\rangle .
$$

In particular, we have $N_{1} \equiv M_{0}$. More significantly, since $N_{0}$ is $\delta$ saturated, both $N_{1}$ and $M_{0}$ are also $\delta$-saturated. Since $N_{1}$ and $M_{0}$ have the same power $\delta$, by the uniqueness of saturated models, we have $N_{1} \cong M_{0}$. From these facts we can draw the following fundamental result: 
(4) If $M^{\prime}=\left\langle A^{\prime}, U^{\prime}, R^{\prime}, S^{\prime}, \cdots\right\rangle$ is any model which can be elementarily embedded into $M_{0}$ by an isomorphic embedding which maps $U^{\prime}$ onto $U_{0}$, then $M^{\prime}$ has a proper elementary extension $M^{\prime \prime}=\left\langle A^{\prime \prime}, U^{\prime \prime}, R^{\prime \prime}, S^{\prime \prime}, \cdots\right\rangle$ such that

$$
U^{\prime}=U^{\prime \prime}, \quad R^{\prime}=R^{\prime \prime}, \quad \text { and } \quad M^{\prime \prime} \cong M_{0} .
$$

We shall define by transfinite induction a sequence of models,

$$
\begin{array}{r}
M_{0}=\left\langle A_{0}, U_{0}, R_{0}, S_{0}, \cdots\right\rangle, \cdots, M_{\nu}=\left\langle A_{\nu}, U_{0}, R_{0}, S_{\nu}, \cdots\right\rangle, \cdots, \\
\quad \text { for every ordinal } \nu<\delta^{+},
\end{array}
$$

satisfying the following: for every $\nu<\delta^{+}$we have

(5) $M_{0} \cong M_{\lambda}$ for every ordinal $\lambda \leqq \nu$, and $M_{*}$ is a proper elementary submodel of $M_{\lambda}$ for all ordinals $\kappa, \lambda$ such that $\kappa<\lambda \leqq \nu$.

Let $\mu$ be an ordinal such that $0<\mu<\delta^{+}$. Assume that $M_{\nu}$ has been defined for all $\nu<\mu$ and that (5) holds for all $\nu<\mu$. If $\mu=\nu^{+}$for some $\nu$, then by (4) we can easily find a model $M_{\mu}=\left\langle A_{\mu}, U_{0}, R_{0}, S_{\mu}, \cdots\right\rangle$ such that

$M_{0} \cong M_{\mu}$ and $M_{\nu}$ is a proper elementary submodel of $M_{\mu}$.

Hence (5) will hold with $\mu$ in the place of $\nu$.

Suppose that $\mu$ is a limit ordinal. Let

$$
M^{\prime}=\left\langle A^{\prime}, U_{0}, R_{0}, S^{\prime}, \cdots\right\rangle=\underset{\lambda<\mu}{\bigcup} M_{\lambda} .
$$

Clearly $M^{\prime}$ is a proper elementary extension of each $M_{\lambda}, \lambda<\mu$, and $\left|A^{\prime}\right|=\delta$. We prove that

(6) $M^{\prime}$ is elementarily embeddable into $M_{0}$ in such a way that $U_{0}$ is mapped onto $U_{0}$.

The proof of (6) will take some time.

Let $a \in\left(U_{0}\right)^{8}$ and $a^{\prime} \in\left(A^{\prime}\right)^{8}$ be enumerations of $U_{0}$ and $A^{\prime}$, respectively. In what follows the letter $\eta$ shall range over limit ordinals including 0 . We shall construct by a second transfinite induction two sequences $b \in\left(A_{0}\right)^{\delta}$ and $b^{\prime} \in\left(A^{\prime}\right)^{\delta}$ such that for every $\nu<\delta$,

$$
\left(M_{0}, b \uparrow \nu+1\right) \equiv\left(M^{\prime}, b^{\prime} \uparrow \nu+1\right)
$$

$$
\begin{aligned}
& \text { if } \nu=\eta+2 k \text { with } k<\omega \text {, then } b_{\nu}^{\prime}=a_{\eta+k}^{\prime}, \text { and } \\
& \text { if } \nu=\eta+2 k+1 \text { with } k<\omega \text {, then } b_{\nu}=a_{v+k} \text {. }
\end{aligned}
$$

Let $\lambda<\delta$ and let us suppose that the sequences $b$ and $b^{\prime}$ have been 
defined for each $\nu<\lambda$ and that (7) and (8) hold for each $\nu<\lambda$. From (7), we immediately have

$$
\left(M_{0}, b \uparrow \lambda\right) \equiv\left(M^{\prime}, b^{\prime} \uparrow \lambda\right) .
$$

Assume that $\lambda=\eta+2 k$ with $k<\omega$. We define $b_{\lambda}^{\prime}=a_{\eta+k}^{\prime}$. Let $C$ be the set of all formulas $\phi\left(v_{0}\right)$ in the language of $\left(M^{\prime}, b^{\prime} \uparrow \lambda\right)$ with at most one free variable $v_{0}$ such that

$$
\left(M^{\prime}, b^{\prime} \mid \lambda\right) \vDash \phi\left(b_{\lambda}^{\prime}\right) .
$$

$C$ is finitely satisfiable in $\left(M^{\prime}, b^{\prime} \uparrow \lambda\right)$. Hence, by (9), $C$ is finitely satisfiable in $\left(M_{0}, b \uparrow \lambda\right)$. Since $M_{0}$ is $\delta$-saturated and $|C|<\delta$, we have $C$ is simultaneously satisfiable in $\left(M_{0}, b\lceil\lambda)\right.$ by some element $e \in A_{0}$. We define $b_{\lambda}=e$. It follows that

$$
\left(\left(M_{0}, b\lceil\lambda), b_{\lambda}\right) \equiv\left(\left(M^{\prime}, b^{\prime} \uparrow \lambda\right), b_{\lambda}^{\prime}\right)\right.
$$

and

$$
\left(M_{0}, b \uparrow \lambda+1\right) \equiv\left(M^{\prime}, b^{\prime} \uparrow \lambda+1\right) .
$$

Hence (7) and (8) hold with $\lambda$ in place of $\nu$.

Assume that $\lambda=\eta+2 k+1$ with $k<\omega$. We define $b_{\lambda}=a_{\eta+k}$. Let $D$ be the set of all formulas $\phi\left(v_{0}\right)$ in the language of $\left(M_{0}, b \uparrow \lambda\right)$ (the same language as that of $\left(M^{\prime}, b^{\prime} \uparrow \lambda\right)$ ) with at most one free variable $v_{0}$ such that

$$
\left(M_{0}, b \uparrow \lambda\right) \vDash \phi\left(b_{\lambda}\right) \text {. }
$$

Notice that the formula $U\left(v_{0}\right) \in D . D$ is finitely satisfiable in $\left(M_{0}, b\lceil\lambda)\right.$, so by (9), $D$ is finitely satisfiable in $\left(M^{\prime}, b^{\prime} \uparrow \lambda\right)$. We shall prove that

(10) $D$ is simultaneously satisfiable in $\left(M^{\prime}, b^{\prime} \uparrow \lambda\right)$ by some element $e \in U_{0}$.

Let $S_{\omega}(D)$ denote the set of all finite subsets of $D$. Notice that $\left|S_{\omega}(D)\right|$ $<\delta$. Consider a set $d \in S_{\omega}(D)$. Let $\phi_{d}\left(v_{0}\right)$ be the conjunction of all the formulas in $d \cup\left\{U\left(v_{0}\right)\right\}$. It follows that

$$
\left(M^{\prime}, b^{\prime}\lceil\lambda) \vDash\left(\exists v_{0}\right) \phi_{d}\left(v_{0}\right) .\right.
$$

Remember that $\phi_{d}$ is a formula in the language of $\left(M^{\prime}, b^{\prime} \uparrow \lambda\right)$, hence there are a finite number of the terms of the sequence $b^{\prime} \uparrow \lambda$, say $b_{p_{1}}, \cdots, b_{p_{1}}$, such that

$$
M^{\prime} \vDash\left(\exists v_{0}\right) \phi_{d}\left(v_{0}, b_{v_{1}}, \cdots, b_{v_{n}}\right),
$$

where $\phi_{d}\left(v_{0}, v_{1}, \cdots, v_{n}\right)$ is some formula of $L_{1}$. Let $\nu_{d}<\mu$ be an ordinal such that 


$$
b_{p_{1}}, \cdots, b_{v_{n}} \in A_{v_{d}} .
$$

Then since $M_{v_{d}} \prec M^{\prime}$, we have

$$
M_{v_{d}} \vDash\left(\exists v_{0}\right) \phi_{d}\left(v_{0}, b_{v_{1}}, \cdots, b_{v_{n}}\right) .
$$

We can now select, for each $d \in S_{\omega}(D)$, the elements $b_{v_{1}}, \cdots, b_{n_{n}}$, the ordinal $\nu_{d}$, and an element $e_{d} \in U_{0}$ such that

$$
M_{v_{d}} \vDash \phi_{d}\left(e_{d}, b_{v_{1}}, \cdots, b_{p_{n}}\right) \text {. }
$$

Notice that if $d \subset d^{\prime} \in S_{\omega}(D)$, then

$$
\vdash \phi_{d^{\prime}}\left(v_{0}\right) \rightarrow \phi_{d}\left(v_{0}\right)
$$

and since $e_{d^{\prime}} \in U_{0} \subset A_{v_{d}}$,

$$
M_{v_{d}} \vDash \phi_{d}\left(e_{d^{\prime}}, b_{p_{1}}, \cdots, b_{n_{n}}\right) \text {. }
$$

Again let $d \in S_{\omega}(D)$ and consider the set of conditions

$$
\begin{aligned}
E_{d}= & \left\{\boldsymbol{R}\left(v_{0}, e_{d^{\prime}}\right): d \subset d^{\prime} \in S_{\omega}(D)\right\} \\
& \cup\left\{\forall t\left(R\left(v_{0}, t\right) \rightarrow \phi_{d}\left(t, b_{v_{1}}, \cdots, b_{v_{n}}\right)\right)\right\} .
\end{aligned}
$$

Using the fact that $M_{r_{d}}$ is a model of $T^{\prime}$, by (3), we see that every finite subset of $E_{d}$ is satisfiable in $M_{v_{d}}$. Since $M_{v_{d}}$ is $\delta$-saturated and $\left|E_{d}\right|<\delta$, we have that $E_{d}$ is simultaneously satisfiable in $M_{v_{d}}$. This means we can select for each $d \in S_{\omega}(D)$ an element $f_{d} \in U_{0}$ such that

$$
M_{v_{d}} \vDash \forall t\left(R\left(f_{d}, t\right) \rightarrow \phi_{d}\left(t, b_{v_{1}}, \cdots, b_{v_{n}}\right)\right),
$$

and

$$
M_{v_{d}} \vDash R\left(f_{d}, e_{d^{\prime}}\right) \quad \text { for all } d^{\prime} \text { such that } d \subset d^{\prime} \in S_{\omega}(D) \text {. }
$$

Consider now a third set of conditions,

$$
F=\left\{R\left(f_{d}, v_{0}\right): d \in S_{\omega}(D)\right\} .
$$

If $R\left(f_{d_{1}}, v_{0}\right), \cdots, R\left(f_{d_{m}}, v_{0}\right) \in F$, then letting $e^{\prime}=e_{\left(d_{1} \cup \cdots \cup d_{m}\right)}$, we have by (13),

$$
M_{v_{d_{i}}} \vDash R\left(f_{d_{i}}, e^{\prime}\right) \quad \text { for all } i \text { such that } 1 \leqq i \leqq m .
$$

Since all the elements involved belong to $U_{0}$ and hence to $A_{0}$, we have

$$
M_{0} \vDash R\left(f_{d_{1}}, e^{\prime}\right) \wedge \cdots \wedge R\left(f_{d_{m}}, e^{\prime}\right) .
$$

So $F$ is finitely satisfiable in $M_{0}$. Since $M_{0}$ is $\delta$-saturated and $|F|<\delta$, we have

(14) $F$ is simultaneously satisfiable in $M_{0}$ by some element 


$$
e \in U_{0} \text {. }
$$

It remains to prove (10). It is sufficient to show that

$$
\left(M^{\prime}, b^{\prime} \uparrow \lambda\right) \vDash \phi_{d}(e) \quad \text { for all } d \in S_{\omega}(D) \text {. }
$$

So, let $d \in S_{\omega}(D)$. By (14) we have

$$
M_{0} \vDash R\left(f_{d}, e\right),
$$

and, since $M_{0} \prec M_{v_{d}}$,

$$
M_{v_{d}} \vDash R\left(f_{d}, e\right) .
$$

By (12), we have

$$
M_{v_{d}} \vDash \phi_{d}\left(e, b_{p_{1}}, \cdots, b_{v_{n}}\right),
$$

and, since $M_{v_{d}} \prec M^{\prime}$,

$$
M^{\prime} \vDash \phi_{d}\left(e, b_{p_{1}}, \cdots, b_{p_{n}}\right) .
$$

This means $\left(M^{\prime}, b^{\prime} \backslash \lambda\right) \vDash \phi_{d}(e)$, and (10) is proved. We define $b_{\lambda}^{\prime}=e$. It is now immediate that conditions (7) and (8) again hold with $\lambda$ in place of $\nu$.

This completes the second transfinite induction and (7) and (8) hold for all $\nu<\delta$. It follows that

$$
\begin{aligned}
& \left(M_{0}, b\right) \equiv\left(M^{\prime}, b^{\prime}\right), \\
& \text { the range of } b^{\prime} \text { is } A^{\prime} \text {, and } \\
& \text { the range of } b \text { includes } U_{0} .
\end{aligned}
$$

Let $h$ be the mapping

$$
h: b_{\dot{\prime}}^{\prime} \rightarrow b, \quad \text { for all } \nu<\delta .
$$

It is a simple matter to verify that $h$ is a one-to-one elementary embedding of $M^{\prime}$ into $M_{0}$ such that $U_{0}$ is mapped onto $U_{0}$. So (6) has been proved. Using (6) and (4), we can again find a model $M_{\mu}$ $=\left\langle A_{\mu}, U_{0}, R_{0}, S_{\mu}, \cdots\right\rangle$ such that (5) holds with $\mu$ in place of $\nu$. This completes our original transfinite induction and (5) now holds for every $\nu<\delta^{+}$.

Let $M=\mathrm{U}_{p<0^{+}} M_{\eta}$. It is clear that $M$ is a model of $T^{\prime}$ of power $\delta^{+}$ with the set $U_{0}$ of power $\delta$. Throwing away the superfluous relation $R_{0}$, the resulting model is a model of $T$. Hence $T$ admits $\delta^{+}, \delta$ and the theorem is proved.

REMARKs. (1) The restriction to countable theories $T$ is not necessary. We can establish, again assuming the generalized continuum hypothesis, that: If $T$ has $\kappa \geqq \omega$ symbols and $T$ admits $\alpha>\beta \geqq \omega$, then $T$ admits every pair of cardinals $\delta^{+}, \delta$ where $\delta$ is a regular infinite 
cardinal greater than $\kappa$. This result can be proved on either one of the following two ways. One can use the fact that there exist $\delta$-saturated models of power $\delta$ in any theory $T$ with $\kappa$ symbols and with an infinite model, provided that $\delta$ is regular infinite and greater than $\kappa$. The argument then parallels the argument given in the theorem. One can also use the fact that, using the theorem, every countable subtheory of $T$ will admit the pair $\delta^{+}, \delta$ for a regular $\delta$. If $\delta>\kappa$, then by an easy ultraproduct argument (see [1], for instance) we can show that $T$ admits the pair $\delta^{+}, \delta$. Curiously, neither one of the above arguments will prove the very first case, namely, $T$ admits $\kappa^{+}, \kappa$ if $\kappa$ is itself regular. Recently, by using the fact that the proof of our theorem involves saturated models, Vaught has shown (private communication, unpublished) that the missing case can be proved. One intriguing problem of the same type remains open. Suppose $T$ has $\kappa^{++}$symbols and $T$ admits $\kappa^{+}, \kappa$. Then must $T$ admit $\kappa^{++}, \kappa^{+}$?

(2) As the reader can see, the proof of the theorem involves the construction of a tower of models

$$
M_{0}, \cdots, M_{\nu}, \cdots \quad \text { for } \nu<\delta^{+} \text {, }
$$

with the fixed set $U_{0}$. This is the basic idea used by Vaught in his first proof for the case $\delta=\omega[4]$. The whole trick, and indeed the only trick, of our proof is to show that the process can be continued at the limit ordinals. The introduction of the binary relation $R$ which indexes the finite sets of $U$ is the key to the argument. The trick seems to break down if $\delta$ is a singular cardinal and $M_{0}$ is taken to be a special model of power $\delta$ (in the sense of [4]). Hence, it is still not known if $T$ must admit all pairs $\delta^{+}, \delta$ if $T$ admits some pair $\alpha, \beta$ where $\alpha>\beta \geqq \omega$.

(3) The question also remains open whether this trick, or some similar device, will enable us to show that if $T$ admits $\alpha, \beta$ where $\alpha>\beta^{+}$and $\beta \geqq \omega$, then $T$ must admit all pairs $\delta^{++}, \delta$ where $\delta$ is an infinite regular cardinal. By a simple observation this last result, if provable, surely must require the generalized continuum hypothesis. This is because it follows from the work of Cohen [2] that it is consistent to assume that $2^{\omega}=\omega_{2}$ and, say, $2^{\omega_{2}}=\omega_{3}$. Hence, one can easily construct a theory $T$ which admits $\omega_{2}, \omega$, but which does not admit $\omega_{4}, \omega_{2}$. This observation does not seem to show that the generalized continuum hypothesis is necessary for our theorem.

\section{REFERENCES}

1. C. C. Chang and H. J. Keisler, Applications of ultraproducts of pairs of cardinals to the theory of models, Pacific J. Math. 12 (1962), 835-845. 
2. P. J. Cohen, The independence of the continuum hypothesis, Proc. Nat. Acad. Sci. U. S. A. 50 (1963), 1143-1148; 51 (1964), 105-110.

3. H. J. Keisler, Ultraproducts and saturated models, Indag. Math. 26 (1964), 178186.

4. M. Morley and R. Vaught, Homogeneous universal models, Math. Scand. 11 (1962), 37-57.

5. A. Tarski and R. Vaught, Arithmetical extensions of relational systems, Compositio Math. 13 (1957), 81-102. 313.

6. R. Vaught, Models of complete theories, Bull. Amer. Math. Soc. 69 (1963), 299-

7. - A Löwenheim-Skolem theorem for cardinals far apart, Proceedings of Symposium on Model Theory, Berkeley, Calif., June-July, 1963 (to appear).

University of California, Los Angeles

\section{A REMARK ON WIENER'S TAUBERIAN THEOREM}

M. KAC

A recent note by Levinson [1] made it seem worthwhile to point out that a weaker version of the Tauberian theorem can be proved in a few lines which is, however, strong enough to provide a proof of the prime number theorem.

Let $K(x) \in L(-\infty, \infty)$ and assume that its Fourier transform obeys the standard condition

$$
\begin{aligned}
\kappa(\xi) & =\int_{-\infty}^{\infty} K(x) e^{i \xi x} d x \\
& \neq 0 \text { for all }-\infty<\xi<\infty .
\end{aligned}
$$

One version of Wiener's Tauberian theorem is the assertion that if $m(y)$ is a bounded measurable function such that for almost all $x$,

$$
\int_{-\infty}^{\infty} K(x-y) m(y) d y=0
$$

then $m(y)=0$ almost everywhere.

The weaker version of the Tauberian theorem is obtained by adding an extra requirement on the function $K(x)$, namely that

$$
x^{2} K(x) \in L(-\infty, \infty) .
$$

Received by the editors October 10, 1964. 